\title{
EL CAPITAL INTELECTUAL Y LA GENERACIÓN DE VALOR EN EL SECTOR TECNOLÓGICO
}

\section{Intellectual Capital and the Generation of Value in the Technology Sector}

\author{
Laura Cayetano Cardona ${ }^{1}$ \\ María Luisa Saavedra García²
}

\section{RESUMEN}

El capital intelectual es un factor clave dentro de la empresa, su correcta gestión impulsa la generación de valor y la creación de ventajas competitivas, razón por la cual es necesario su análisis e incorporación en las cifras financieras para la correcta toma de decisiones. En esta investigación se utilizó el método de caso simple, recolectando datos de una importante empresa del sector tecnológico: Samsung Electronics, realizando una investigación de tipo mixto con alcance correlacional, utilizando la aplicación de los modelos Skandia, VAIC (Value Added Intelectual Coefficient) y EVA (Economic, Value, Added). Los principales hallazgos de este trabajo permitieron determinar que es posible calcular el valor monetario del capital intelectual utilizando el modelo Skandia, así también que existe una importante relación entre el valor del capital intelectual y el valor de la empresa calculado con EVA, lo que demuestra la importancia de gestionar correctamente el capital intelectual para generar valor en la empresa. Así también, se encontró que existe relación entre el valor del capital intelectual y el VAIC, lo cual implica la necesidad de gestionar correctamente el capital intelectual para obtener eficiencia en el uso de este.

\footnotetext{
${ }^{1}$ Maestra en Finanzas Corporativas, FCA-UNAM y consultora independiente. Correo electrónico: laura.cayetano.c@gmail.com

${ }^{2}$ Contadora Pública por la Universidad de San Martín de Porres de Lima-Perú, especialista en Finanzas, Maestra y Doctora en Administración por la FCA/UNAM. Obtuvo la medalla de plata "Gabino Barreda" por sus estudios de maestría, y la medalla "Alfonso Caso" por sus estudios de doctorado. Asimismo, obtuvo el "Premio de investigación Financiera IMEF 2002"; el reconocimiento de académico certificado por ANFECA 2003; el reconocimiento al mérito académico ANFECA 2003 “Arturo Elizundia Charles" (Premio a la docencia en Contaduría y Administración), y el primer lugar del Premio Nacional de Tesis de grado "ANFECA 2003". Correo electrónico: Isaavedra@fca.unam.mx
} 
Palabras clave: Capital intelectual, EVA, VAIC, Skandia, sector tecnológico. Clasificación JEL: G12, G13, J24, L96.

\section{ABSTRACT}

The intellectual capital is a key factor within the company, its correct management drives the generation of value and the creation of competitive advantages, its analysis and incorporation into the financial figures are necessary in order to make correct decisions. This investigation uses the simplest case method, it collects data from an important company in the technology sector, Samsung Electronics. A mixed-type investigation with correlational scope is conducted, using the Skandia, VAIC (Value Added Intellectual Coefficient) and EVA (Economic, Value, Added) models. The main findings allowed to determine that it is possible to calculate the monetary value of intellectual capital using the Skandia model and there is an important relationship between the intellectual capital value and the company value calculated with EVA which demonstrates the correctly managing intellectual capital is important to generate value in the company. Likewise, there is a relationship between intellectual capital value and the VAIC which implies the need to correctly manage intellectual capital to obtain efficiency in its use.

Keywords: Value creation, intellectual capital, EVA, VAIC, Skandia, technology sector. JEL Classification: G12, G13, J24, L96.

\section{Introducción}

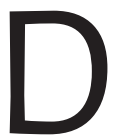
emuner, Saavedra y Camarena (2017) señalan que autores como Steward, Edvinsson y Malone, Sveiby y Pulic han argumentado que las medidas tradicionales de rendimiento de una empresa, que se basan en los principios de contabilidad convencionales, pueden ser incorrectas. A su vez, Adam (2005) menciona que los informes financieros tradicionales muestran en forma limitada el valor de la empresa porque el capital contable sólo refleja información pasada, lo que lleva a reconsiderar si dichas medidas son suficientes o es necesario tomar en cuenta otros elementos que brinden información valiosa y que la hagan más verídica y útil. Aunado a esto Joya, Gámez, Ortiz y Gálvez (2015) indican que los activos intangibles no son reconocidos como elementos que influyen en el valor contable real que posee una organización, por lo cual no son reflejados en 
los estados financieros y en los informes de gestión que constituyen la base del proceso de toma de decisiones. Por otro lado, "el capital intelectual se entiende como el conjunto de activos intangibles (basados en el conocimiento) que forman una empresa en un momento determinado y que permiten conseguir una ventaja competitiva a través del conocimiento" (Cegarra y Jiménez, 2002, p19). Tomando en cuenta las aseveraciones anteriores se entiende que el capital intelectual es una fuente generadora de ventajas estratégicas y que es relevante que se incluya como parte de esas medidas de rendimiento que puedan dar un fundamento más sólido en la toma de decisiones.

No obstante, el capital intelectual no es considerado como parte del valor contable de la empresa: en un estudio realizado en empresas españolas Villacorta (2003) encontró que "las empresas ofrecen la información voluntaria que más les interesa para sus propias motivaciones y, sin embargo, los usuarios de la contabilidad financiera necesitan una información más amplia y comparable sobre el capital intelectual" (p. 365). Joya et al. (2015) concluyeron en su investigación que "México presenta serias limitaciones en la gestión de su capital intelectual. Constituye una necesidad que se desarrollen políticas y estrategias a los diferentes niveles gubernamentales que estimule a los académicos y empresarios mexicanos en el diseño e implementación de metodologías y procedimientos adecuados a sus realidades" (p. 93); es decir, no es un problema limitado a ciertos países, sino que al no existir normatividad que obligue a las empresas a revelar información, no lo hacen o lo hacen de acuerdo con sus propios criterios.

Desde el punto de vista financiero, uno de los objetivos de la empresa es la generación de valor y para lograrlo debe crear estrategias de acuerdo con sus necesidades y la realidad; pero es indispensable contar con la información suficiente, tanto histórica, proveniente de los estados financieros, como del capital intelectual, para tener mayor certeza de que las decisiones tomadas para impulsar la generación de valor son las correctas.

El objetivo de esta investigación es determinar el valor del capital intelectual mediante la utilización de modelos de medición tanto de su valor como de su eficiencia y establecer su relación con el valor económico agregado (EVA) en las empresas. Esta investigación consta de cuatro partes, la siguiente parte que se refiere a la revisión de la literatura, en la cual se abordan temas como el concepto de capital intelectual, sus componentes, los modelos o métodos de medición y un panorama del capital intelectual en México, así como del sector tecnológico. La tercera parte se refiere a la metodología utilizada para realizar la recolección y 
análisis de datos. La cuarta parte incluye los hallazgos o resultados derivados del análisis y finalmente se presentan las conclusiones.

\section{Revisión de la literatura}

La eficacia empresarial en este siglo XXI ya no radica en lo importante o el valor de los edificios o maquinaria, sino en activos inmateriales, referidos a la fidelidad de los clientes, a la propiedad intelectual, al potencial innovador, a los conocimientos técnicos y especializados del personal (Larios, 2010). Todos esos activos inmateriales se concentran en lo que se conoce como capital intelectual, pero ¿qué es capital intelectual? Brooking (1997), Sveiby (1997) y Mena, Vásconez y Carguaytongo (2017) mencionan que es un conjunto o combinación de activos intangibles que incluyen como lo indican Edvinsson y Malone (1997), Stewart (1997), Kristandl y Bontis (2007), Sarur (2013) y Archibold y Escobar (2015); el conocimiento originado por el recurso humano, las relaciones de la empresa con externos como los clientes y los proveedores, la propiedad intelectual como marcas y patentes, además de recursos estratégicos organizativos los cuales según Nevado y López (2016), Hernando y Cervera (2018) son fuentes generadoras de valor y de beneficios a las empresas, además de que estos pueden ser en el presente o a futuro.

Los componentes del capital intelectual se clasifican de diferentes maneras, como se muestra en el cuadro 1, donde se puede observar que no difieren mucho y hacen referencia a factores muy similares, los cuales pueden encontrarse de forma general en cualquier entidad; es decir que en cada empresa existe capital intelectual y, por tanto, posee una fuente generadora de valor $y$ de ventajas competitivas. En ello radica la importancia de identificarlo y de gestionarlo de la forma correcta con la finalidad de maximizar sus beneficios.

Sin embargo, los componentes del capital intelectual al tener una naturaleza intangible tienden a ser subjetivos y por eso se presenta una complicación al momento de su identificación al igual que en su medición, razón por la cual se han creado propuestas de medición, tanto para identificar su valor como para determinar su eficiencia dentro de la empresa. El elegir un modelo dependerá del objetivo que se quiere alcanzar al momento de medir; de ahí la importancia de que antes de realizarlo se identifique la finalidad de medir quiénes están interesados en la información y el tipo de empresa para la cual se hará dicho análisis, entre otras cosas. En este trabajo se mencionarán algunos de los modelos y se abundará más en aquellos que son más conocidos y que se ajustan más a las necesidades 
de esta investigación; no obstante, estos no limitan el uso de otros modelos que puedan adaptarse mejor a los requerimientos de cada empresa. En el cuadro 2 , se muestra una relación de algunos modelos de medición, cuáles son sus componentes y con qué están relacionados, lo que ayudará a decidir utilizar alguno de acuerdo con el objetivo para el que se quiera medir el capital intelectual.

\section{CUADRO 1. COMPONENTES DEL CAPITAL INTELECTUAL}

Autor Componentes del capital intelectual

Activos de mercado: Constituyen el potencial derivado de los bienes inmateriales que guardan relación con el mercado. Se incluyen marcas,

Brooking (1997) clientela y su fidelidad, rentabilidad del negocio, reserva de pedidos, canales de distribución, diversos contratos y acuerdos tales como licencias, franquicias, etc.

\section{Activos de propie-} dad intelectual: Incluyen el knowhow, secretos de fabricación, copyright, patentes y diversos derechos de diseño, así como también las marcas de fábrica y de servicios.

Activos centrados
en el individuo:
Comprenden pericia
colectiva, capacidad
creativa, habilidad
para resolver pro-
blemas, liderazgo y
capacidad empre-
sarial y de gestión
encarnados en los
empleados de la
organización.

Activos centrados Comprenden pericia colectiva, capacidad para resolver problemas, liderazgo y capacidad empresarial y de gestión organización.

\begin{abstract}
Activos de infraestructura: tecnologías, metodologías y procesos que hacen posible el funcionamiento de la organización. La cultura corporativa, metodologías para el cálculo de riesgos, métodos de dirección de una fuerza de ventas, estructura financiera, bases de datos de información sobre mercado o clientes y sistemas de comunicación, tales como el e-mail y sistemas de teleconferencia.
\end{abstract}

\section{Capital Estructural: Los equipos, programas,} bases de datos, estructura organizacional, pa-

Capital humano: Combinación de conociEdvins- mientos, habilidades, inventiva y capacidad son y Malone (1997) de los empleados individuales de la empresa para llevar a cabo la tarea que tienen entre manos. Incluye igualmente los valores de la empresa, su cultura y su filosofía. La empresa no puede ser propietaria del capital humano. tentes, marcas de fábrica y todo lo que forma parte de la capacidad organizacional que sostiene la productividad de sus empleados -en una palabra, todo lo que se queda en la oficina cuando los empleados se van a su casa-. El capital estructural incluye igualmente el capital clientela, las relaciones desarrolladas con los clientes clave. A diferencia del capital humano, el estructural sí puede ser propiedad de la empresa y por tanto se puede negociar.

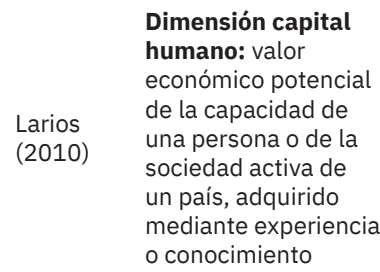

Dimensión capital humano: valor económico potencial una persona o de la sociedad activa de mediante experiencia o conocimiento
Dimensión capital estructural: está constituido por todo aquello que ha fomentado la organización y que la distingue de otras. Está representado por el conocimiento de las personas que se integra a los procesos internos y tecnologías de la organización, a los procedimientos y a los sistemas de trabajo que han desarrollado.
Dimensión capital relacional: es necesario que la empresa defina lo que significa el cliente, lo que necesita y también que conozca aquellas cosas que el cliente necesita, aun cuando ella no las conozca. 
CuAdRo 1. COMPONENTES DEL CAPITAL INTELECTUAL (CONTINUACIÓN)

\begin{tabular}{|c|c|c|c|c|}
\hline Autor & & Componentes de & sapital intelectual & \\
\hline $\begin{array}{l}\text { Saave- } \\
\text { dra y } \\
\text { Saavedra } \\
(2012)\end{array}$ & $\begin{array}{l}\text { Capital humano: } \\
\text { formación, habilida- } \\
\text { des para el trabajo y } \\
\text { experiencia. }\end{array}$ & $\begin{array}{l}\text { Capital estructural: } \\
\text { tecnología, métodos } \\
\text { y procesos, sistemas } \\
\text { informáticos y de } \\
\text { comunicación, bases } \\
\text { de datos, estructu- } \\
\text { ra organizacional, } \\
\text { procesos y procedi- } \\
\text { mientos. }\end{array}$ & $\begin{array}{l}\text { Capital relacional: } \\
\text { clientes, provee- } \\
\text { dores, accionistas, } \\
\text { acreedores. }\end{array}$ & $\begin{array}{l}\text { Capital intelectual } \\
\text { registrado: patentes, } \\
\text { secreto industrial, } \\
\text { derechos de diseño, } \\
\text { marcas. }\end{array}$ \\
\hline
\end{tabular}

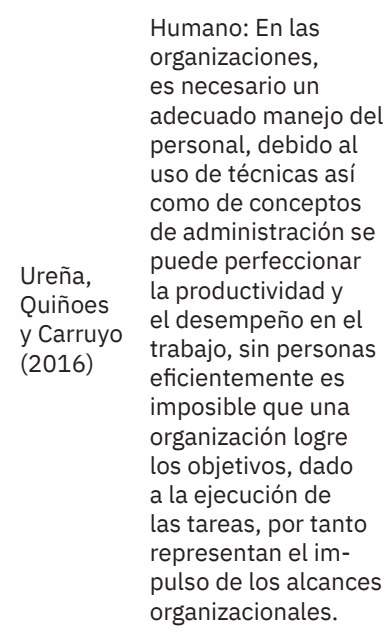

Estructural: incluye factores tales como calidad y alcance de los sistemas de información, imágenes de la empresa, base de datos patentadas, conceptos organizacionales y documentación, renglones tradicionales como propiedad intelectual, incluyendo patentes sí como marcas o derechos de autor.

\begin{abstract}
Clientelar: el capital clientelar se considera como una categoría separada, equivalente a capital estructural y humano, es decir, es una idea interesante y sugiere que las relaciones de una empresa con sus clientes son distintas de su trato con los empleados y los socios estratégicos, y que esta relación es e importancia absolutamente central para el valor de la empresa.
\end{abstract}

Fuente: elaborado con base en Brooking (1997), Edvinsson y Malone (1997), Larios (2010),

Saavedra y Saavedra (2012) y Ureña, Quiñoes y Carruyo (2016) 
CuAdro 2. Modelos de MEdición del CAPITAL INTELECTUAL, COMPONENTES Y RELACIÓN

\begin{tabular}{|c|c|c|c|}
\hline Modelo & Autor & Componentes & Relación \\
\hline \multirow{5}{*}{$\begin{array}{l}\text { Modelo de las Cinco } \\
\text { Fases de Nonaka y } \\
\text { Takeuchi }\end{array}$} & \multirow{5}{*}{$\begin{array}{l}\text { (Nonaka y } \\
\text { Takeuchi, 1995) }\end{array}$} & Compartir el conocimiento tácito & \multirow{5}{*}{$\begin{array}{l}\text { Conocimiento tácito y } \\
\text { explicito }\end{array}$} \\
\hline & & Crear Conceptos & \\
\hline & & Justificar los Conceptos & \\
\hline & & Construir arquetipos & \\
\hline & & Expandir el conocimiento & \\
\hline \multirow{4}{*}{$\begin{array}{l}\text { Modelo de Canadian } \\
\text { Imperial Bank }\end{array}$} & \multirow{4}{*}{$\begin{array}{l}\text { (Hubert } \\
\text { Saint-Onge, } \\
\text { 1996) }\end{array}$} & Capital humano & \multirow{4}{*}{$\begin{array}{l}\text { Aprendizaje Organi- } \\
\text { zacional }\end{array}$} \\
\hline & & Capital estructural & \\
\hline & & Capital clientes & \\
\hline & & Capital Financiero & \\
\hline \multirow{3}{*}{$\begin{array}{l}\text { Modelo Universidad de } \\
\text { West Ontario }\end{array}$} & \multirow{3}{*}{ (Bontis, 1996) } & Capital humano & \multirow{3}{*}{ Desempeño } \\
\hline & & Capital estructural & \\
\hline & & Capital relacional & \\
\hline \multirow{3}{*}{$\begin{array}{l}\text { Modelo de Dow Che- } \\
\text { mical }\end{array}$} & \multirow{3}{*}{ (Dow, 1998) } & Capital humano & \multirow{3}{*}{ Valor } \\
\hline & & Capital organizacional & \\
\hline & & Capital de clientes & \\
\hline \multirow{5}{*}{$\begin{array}{l}\text { Modelo de Dirección } \\
\text { Estratégica por com- } \\
\text { petencia }\end{array}$} & \multirow{5}{*}{ (Bueno, 1998) } & Capital intangible & \multirow{5}{*}{ Dirección } \\
\hline & & Capital humano & \\
\hline & & Capital organizativo & \\
\hline & & Capital tecnológico & \\
\hline & & Capital relacional & \\
\hline \multirow{3}{*}{$\begin{array}{l}\text { Modelo de Estructura } \\
\text { de Capital Intelectual } \\
\text { "Intelect" }\end{array}$} & \multirow{3}{*}{$\begin{array}{l}\text { (EUROFORUM, } \\
\text { 1998) }\end{array}$} & Capital humano & \multirow{3}{*}{ Presente-Futuro } \\
\hline & & Capital estructural & \\
\hline & & Capital relacional & \\
\hline \multirow{4}{*}{ Modelo Skandía } & \multirow{4}{*}{$\begin{array}{l}\text { (Edvinsson y } \\
\text { Malone, 1998) }\end{array}$} & Capital Humano & Pasado \\
\hline & & Capital estructural & Presente \\
\hline & & -Capital Clientela & Futuro \\
\hline & & -Capital Organizacional & \\
\hline \multirow{4}{*}{ Modelo Nova } & \multirow{4}{*}{$\begin{array}{l}\text { (Nova Care, } \\
\text { 1999) }\end{array}$} & Capital humano & \multirow{4}{*}{ Crecimiento } \\
\hline & & Capital de organización & \\
\hline & & Capital Social & \\
\hline & & Capital de innovación y aprendizaje & \\
\hline \multirow{5}{*}{$\begin{array}{l}\text { Modelo de Innovation } \\
\text { Intellectual Capabi- } \\
\text { lities Benchmarking } \\
\text { System }\end{array}$} & \multirow{5}{*}{$\begin{array}{l}\text { (IICBS VIEDMA, } \\
\text { 2001) }\end{array}$} & Capacidades & \multirow{5}{*}{ Benchmarking } \\
\hline & & Procesos & \\
\hline & & Productos y servicios & \\
\hline & & Objetivos del proyecto & \\
\hline & & Necesidades de los clientes & \\
\hline
\end{tabular}


Cuadro 2. Modelos de MEdición del Capital INTELECTUAL, COMPONENTES Y RELACIÓN (CONTINUACIÓN)

\begin{tabular}{|c|c|c|c|}
\hline Modelo & Autor & Componentes & Relación \\
\hline \multirow{3}{*}{$\begin{array}{l}\text { Modelo de Valoración y } \\
\text { Gestión }\end{array}$} & \multirow{3}{*}{$\begin{array}{l}\text { (Nevado y López, } \\
\text { 2002) }\end{array}$} & Capital humano & Valoración \\
\hline & & Capital estructural & Gestión \\
\hline & & Capital no explicitado & Evolución \\
\hline
\end{tabular}

Fuente: elaborado con base en González (2009), Arango, Molina y Zapata (2010), Nevado y López (2016).

\subsection{Modelo Skandia}

El modelo Skandia tiene su origen en la compañía sueca de seguros y servicios financieros Skandia, de ahísu nombre, fue presentado por Leif Edvinsson y Michael S. Malone y es uno de lo más usados por su capacidad para medir el capital intelectual.

El modelo explica que el capital intelectual es la suma de dos clases de factores:

\section{El capital humano}

El capital estructural: a su vez se compone de dos elementos:

- Capital Clientela: Good Will

- Capital Organizacional: competencia sistematizada, empacada y codificada de una organización. Este capital tiene otras dos vertientes:

1. Capital Innovación: capacidad de renovación y los resultados de la innovación en forma de derechos comerciales protegidos, propiedad intelectual y otros activos intangibles.

2. Capital proceso: Conocimiento práctico que se utiliza en la creación continúa de valor.

De acuerdo con Bontis (2001), el modelo Navigator utiliza 112 índices los cuales emplean conteos directos, montos en dólares, porcentajes e incluso resultados de encuestas. También indica que Edvinsson y Malone fomentan que los conteos directos se comparen con otros conteos directos para producir proporciones o transformarse en dinero, dejando solo dos tipos de medición. Las medidas monetarias se combinan utilizando una ponderación predeterminada 
para producir un valor de capital intelectual total para la organización. Los porcentajes, que pueden considerarse medidas de incompletitud, se pueden combinar para producir el coeficiente de eficiencia de capital intelectual que captura la velocidad, la posición y la dirección de la organización. La fórmula que Skandia propone para medir el capital intelectual es la siguiente (Demuner, Saavedra y Camarena, 2017):

\section{Capital Intelectual Organizativo $=\mathrm{iC}$ donde $\mathrm{i}=(\mathrm{n} / \mathrm{x})$}

Donde:

C: es el valor del capital intelectual en unidades monetarias.

i: es el coeficiente de eficiencia con que la organización está utilizando dicho capital. $\mathrm{n}$ : es igual a la suma de los valores decimales de los nueve índices de eficiencia propuestos por Edvinsson y Malone.

x: es el número de esos índices

Bontis (2001) indica que el modelo de Skandia es particularmente impresionante al reconocer el papel del capital del cliente en la creación de valor para una organización y como ha cambiado la naturaleza misma de las relaciones con los clientes. En el cuadro 3 se presentan algunas investigaciones empíricas donde se ha utilizado el modelo Skandia, principalmente para identificar indicadores específicos del mismo.

CuAdro 3. Estudios EMPÍRICOS DE CI CON EL MOdELO SKANDIA

\begin{tabular}{|c|c|c|c|}
\hline Autor & Variables & Muestra & Resultados \\
\hline $\begin{array}{l}\text { Mejía y Pérez } \\
\text { (2013). }\end{array}$ & $\begin{array}{l}\text { Enfoque financiero } \\
\text { Enfoque clientela } \\
\text { Enfoque proceso } \\
\text { Enfoque humano } \\
\text { Enfoque de renovación } \\
\text { y desarrollo. }\end{array}$ & $\begin{array}{l}\text { Entrevista de exper- } \\
\text { tos ( } 3 \text { investigadores } \\
\text { y } 3 \text { consultores). }\end{array}$ & $\begin{array}{l}\text { Los indicadores que se consideran } \\
\text { más apropiados para la identificación } \\
\text { del capital intelectual en los centros } \\
\text { de investigación seleccionados. }\end{array}$ \\
\hline $\begin{array}{l}\text { Hernández, } \\
\text { Flores y Cortés } \\
\text { (2012). }\end{array}$ & $\begin{array}{l}\text { Capital Humano } \\
\text { Capital Estructural } \\
\text { Capital Relacional. }\end{array}$ & $\begin{array}{l}\text { Estudio de caso, } \\
\text { empresa del sector } \\
\text { automotriz, } 76 \\
\text { trabajadores. }\end{array}$ & $\begin{array}{l}\text { Las unidades organizacionales de } \\
\text { logística, comercial, operaciones y } \\
\text { calidad muestran semejanza en el } \\
\text { capital intelectual. }\end{array}$ \\
\hline $\begin{array}{l}\text { Herrera, Ramírez } \\
\text { y May (2012). }\end{array}$ & $\begin{array}{l}\text { Capital humano indica- } \\
\text { dor estabilidad } \\
\text { Capital humano indica- } \\
\text { dor desempeño } \\
\text { Capital humano indica- } \\
\text { dor renovación. }\end{array}$ & $\begin{array}{l}\text { Caso de estudio, or- } \\
\text { ganización artística, } \\
25 \text { trabajadores. }\end{array}$ & $\begin{array}{l}\text { Propuesta de indicadores para eva- } \\
\text { luar el capital intelectual en organiza- } \\
\text { ciones artísticas. }\end{array}$ \\
\hline
\end{tabular}

Fuente: Demuner, Saavedra y Camarena (2017). 


\subsection{Modelo VAIC}

De acuerdo con Villegas, Hernández y Salazar (2017), el Value Added Intelectual Coefficient (VAIC) estructurado por Pulic en 1998 es una ecuación que mide la cantidady la eficiencia del capital intelectual y el capital empleado que crea valor. Tomando como base esta descripción podemos deducir lo que bien mencionan Demuner, Saavedra y Camarena (2017), por medio de este modelo se puede medir la eficiencia del capital intelectual en la creación de valor.

La figura 1 muestra una descripción del modelo VAIC, como se observa, todo parte del valor agregado que es una de las mayores premisas en las organizaciones. Para lograr llegar a obtener el coeficiente intelectual de valor agregado es necesario tomar datos de los reportes financieros que incluyan datos sobre el capital intelectual y el capital empleado ya que de esta forma se puede obtener la eficiencia de cada uno de estos la cual llevará a determinar, a su vez, la eficiencia en la creación de valor.

Figura 1. DESCRIPCIÓN DEL MODELO VAIC

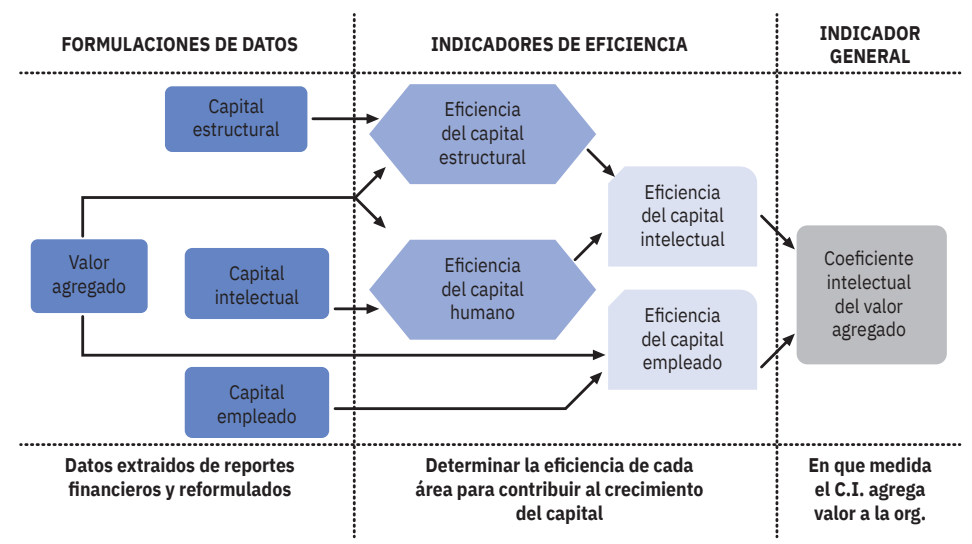

Fuente: (Laing, Dunn y Hughes-Lucas, 2010)

En el cuadro 4, se observan los resultados de los trabajos empíricos que han utilizado el modelo VAIC para medir la eficiencia del capital intelectual, donde la principal bondad es que logra identificar como contribuye cada componente del capital intelectual al valor agregado. 
CuAdro 4. ESTUdios EMPÍRICOS DE CI CON EL MOdELO VAIC

\begin{tabular}{|c|c|c|c|}
\hline Autor & Variables & Muestra & Resultados \\
\hline $\begin{array}{l}\text { Dimitrios } \\
(2004)\end{array}$ & $\begin{array}{l}\text { Rendimiento de CI } \\
\text { Valor agregado. }\end{array}$ & 141 bancos de Japón. & $\begin{array}{l}\text { El CI contribuye al valor agregado } \\
85 \% \text {, el capital físico contribuye } 15 \% \text {. }\end{array}$ \\
\hline Goh (2005). & $\begin{array}{l}\text { Rendimiento de CI y } \\
\text { valor agregado. }\end{array}$ & 16 bancos de Malasia. & $\begin{array}{l}\text { El CI contribuye al valor agregado } \\
99.5 \% \text {, el capital físico contribuye } \\
0.5 \% \text {. }\end{array}$ \\
\hline $\begin{array}{l}\text { Abdulsalam, } \\
\text { Al-Qaheri y } \\
\text { Al-Khayyat } \\
\text { (2011). }\end{array}$ & $\begin{array}{l}\text { Rendimiento de CI y } \\
\text { valor agregado. }\end{array}$ & 8 bancos de Kuwait. & $\begin{array}{l}\text { El CI contribuye al valor agregado } \\
99.5 \% \text {, el capital físico contribuye } \\
0.5 \% \text {. }\end{array}$ \\
\hline $\begin{array}{l}\text { Joshi, Cahill, } \\
\text { Sidhu y Kansal } \\
\text { (2013). }\end{array}$ & $\begin{array}{l}\text { Rendimiento de CI } \\
\text { Rendimiento finan- } \\
\text { ciero. }\end{array}$ & $\begin{array}{l}\text { Sector financiero de } \\
\text { Australia. }\end{array}$ & $\begin{array}{l}\text { El CI contribuye al valor agregado } \\
95 \% \text {, el capital físico contribuye } 5 \% \text {. }\end{array}$ \\
\hline $\begin{array}{l}\text { Ulum, Ghozali } \\
\text { y Purwanto } \\
\text { (2014). }\end{array}$ & $\begin{array}{l}\text { Rendimiento de CI y } \\
\text { valor agregado. }\end{array}$ & $\begin{array}{l}10 \text { bancos de Indo- } \\
\text { nesia. }\end{array}$ & $\begin{array}{l}\text { El CI contribuye al valor agregado } \\
89 \% \text {, el capital físico contribuye } 11 \% \text {. }\end{array}$ \\
\hline $\begin{array}{l}\text { Ozkan, Cakan y } \\
\text { Kayacan (2016). }\end{array}$ & $\begin{array}{l}\text { Rendimiento de CI, } \\
\text { valor agregado, ROA. }\end{array}$ & 44 bancos en Turkia. & $\begin{array}{l}\text { El CI contribuye al valor agregado } \\
93 \% \text {, el capital físico contribuye } 7 \% \text {. }\end{array}$ \\
\hline
\end{tabular}

Fuente: Demuner, Saavedra y Camarena (2017).

\subsection{Modelo de Economic Value Added (EVA)}

El modelo Economic Value Added, más conocido como EVA, es un índice financiero que incorpora el cálculo del costo de los recursos que únicamente fueron utilizados para generar utilidades, proporcionando una medida del valor de una empresa como el resultado del beneficio neto después de impuestos menos el correspondiente cargo por el costo de capital que se encuentra invertido en la compañía (Cázares, Saavedra y Tapia), de ahí la importancia de establecer como se relaciona con el valor del capital intelectual y su eficiencia.

Los métodos para calcular el EVA son (Saavedra, 2004):

\section{Método Diferencial (de Spread)}

El spread es la diferencia entre la tasa de rendimiento del capital y el costo del capital promedio ponderado. Para obtener el EVA, se debe multiplicar et este diferencial por el capital invertido, así se tiene: 


$$
E V A=(R-C C P P) * \text { capital invertido }
$$

\section{Método residual}

Para calcular el EVA con este método se debe obtener la utilidad operativa neta después de impuestos (UNODI), al que se debe restar el cargo por el uso del capital (Capital invertido* $\mathrm{CPPC}$ ), así se tiene:

$$
E V A=U N O D I-(\text { Capital invertido * CCPP })
$$

Los investigadores latinoamericanos han realizado una serie de aplicaciones de este modelo, con el fin de determinar la creación o destrucción de valor en las mismas intentando encontrar los conductores de valor de las empresas. En el cuadro 5 , se presenta a modo de resumen los principales trabajos encontrados.

\begin{tabular}{|c|c|c|}
\hline Autor/Año & País & Hallazgo \\
\hline Matamala y Sagardía (2002) & Chile & $\begin{array}{l}\text { Sólo ocho de los } 36 \text { sectores estudiados crearon valor } \\
\text { en el período 2000-2001. }\end{array}$ \\
\hline Martínez \& López (2003) & Colombia & $\begin{array}{l}\text { Se analizaron las fusiones de empresas desde } 1995 \text { a } \\
\text { 2001, encontrando que toda clase de fusión, genera } \\
\text { valor para las empresas en el corto plazo. }\end{array}$ \\
\hline $\begin{array}{l}\text { Otero, Fernández, \& Rodríguez } \\
\text { (2003) }\end{array}$ & Chile & $\begin{array}{l}\text { Se realiza la adaptación del modelo EVA, para ser } \\
\text { aplicado en el sector asegurador Chileno, encontrando } \\
\text { que son los resultados y la creación de valor los que } \\
\text { orientan las decisiones de activo y pasivo y no otras va- } \\
\text { riables tradicionales como el crecimiento de las ventas } \\
\text { o el beneficio contable. }\end{array}$ \\
\hline Adam (2005) & México & $\begin{array}{l}\text { Se analizaron } 19 \text { empresas que cotizan en la Bolsa } \\
\text { Mexicana de valores, encontrado que una gran propor- } \\
\text { ción de las mismas destruyeron valor, asimismo el EVA } \\
\text { se relaciona con el método de Goodwill. }\end{array}$ \\
\hline Carrillo \& Niño (2006) & Chile & $\begin{array}{l}\text { De las } 35 \text { empresas que se estudiaron en el período de } \\
1994 \text { al 2002, la mayor parte destruyó valor. }\end{array}$ \\
\hline Radi \& Bolívar (2007) & Colombia & $\begin{array}{l}\text { De los } 60 \text { sectores analizados en un período de } 2000 \text { - } \\
2005 \text {, tan sólo el } 32 \% \text { generó valor, mientras que el } \\
68 \% \text { lo destruyó. }\end{array}$ \\
\hline
\end{tabular}

\section{Cuadro 5. Aplicación del EVA en Latinoamérica}




\section{Cuadro 5. Aplicación del EVA en Latinoamérica (CONTINuación)}

\begin{tabular}{lll}
\hline Autor/Año & País & Hallazgo \\
\hline (Díaz (2007) & Venezuela & $\begin{array}{l}\text { El 90\% de las empresas estudiadas confirmaron que } \\
\text { existe una relación entre la optimización del capital y } \\
\text { el EVA. }\end{array}$ \\
\hline Saavedra (2008) & México & $\begin{array}{l}\text { De las 71 empresas que se analizaron, 65\% mostraron } \\
\text { destrucción de valor, inherente al alto costo de capital, } \\
\text { que se tuvo en el período 1991-2000. }\end{array}$ \\
\hline
\end{tabular}

Se estudia el comportamiento de 17 empresas innovadoras por un del sector Alimentos y Bebidas, por el periodo 2000-2008, Se encuentra que las empresas

Rivera y Ruiz (2011) Colombia innovadoras solo crean valor hasta el 2003, a partir de 2004 comienza a destruirlo. Esto obedece principalmente al comportamiento de la eficiencia en el uso de los activos.

\begin{tabular}{|c|c|c|}
\hline $\begin{array}{l}\text { Escobar, Arango, Molina y Arias } \\
\text { (2011) }\end{array}$ & Colombia & $\begin{array}{l}\text { Se aplicó el modelo a una empresa a } 86 \text { empresas } \\
\text { utilizando la información que las mismas presentan } \\
\text { a la Superintendencia de Sociedades por un período } \\
\text { de } 2000-2008 \text {; el principal hallazgo es que existe } \\
\text { una relación inversa entre el EVA y la generación de } \\
\text { utilidades de operación, dato importante dado que } \\
\text { muchas veces se premia la generación de utilidades en } \\
\text { el corto plazo sin tomar en cuenta la sostenibilidad de } \\
\text { la empresa. }\end{array}$ \\
\hline
\end{tabular}

Valerio, Segura y Gutiérrez (2011) Costa Rica

En un grupo de cinco empresas del sector textil se encontró que las empresas fueron capaces de generar valor en los años 2004-2010, al contar con el liderazgo en el mercado.

Se aplicó el modelo a 31 empresas que cotizan en Saavedra y Saavedra (2012) México la Bolsa Mexicana de Valores, encontrando que las empresas de todos los sectores crearon valor en el período 2001-2008, excepto el sector construcción y el sector extractivo los tres primeros años.

\begin{tabular}{|c|c|c|}
\hline $\begin{array}{l}\text { Ramírez, Carbal y Zambrano } \\
\text { (2012) }\end{array}$ & Colo & $\begin{array}{l}\text { Se determinó el valor de una empresa Metalmecánica } \\
\text { por el período } 2006-2010 \text {, encontrando que sólo dos } \\
\text { años genero valor. }\end{array}$ \\
\hline
\end{tabular}

\begin{tabular}{|c|c|c|}
\hline Abreu y Morales (2013) & México & $\begin{array}{l}\text { De una muestra de } 280 \text { empresas manufactureras, } \\
\text { encontraron que sólo } 29 \text { crearon valor, en un período } \\
\text { 1990-2008. }\end{array}$ \\
\hline Téllez, et al. (2017) & México & $\begin{array}{l}\text { Analizaron } 48 \text { empresas que cotizan en la BMV por un } \\
\text { período de } 1998 \text { a 2012, encontrando que la mayoría } \\
\text { de las empresas destruyeron valor y que el EVA no } \\
\text { siempre explica las variaciones de los precios de las } \\
\text { acciones debido a que solo presenta relevancia cuando } \\
\text { la volatilidad económica es baja. }\end{array}$ \\
\hline
\end{tabular}

Fuente: Cazares, Saavedra y Tapia (2019). 
De acuerdo con estos estudios previos, se puede concluir que una alta proporción de las empresas en Latinoamérica destruyen valor, así también estos estudios han sido de gran utilidad para dar a conocer la metodología del EVA y fomentar su aplicación en estos países, lo cual es muy importante puesto que es este modelo proporciona una dimensión real del valor de las empresas.

\section{Método}

Se realizó una investigación de tipo mixto, se recabaron y analizaron datos sobre las variables de los modelos de medición del capital intelectual Skandia y VAIC, así como datos para determinar el valor del EVA. Por otro lado, este estudio es de tipo longitudinal puesto que se analizaron datos de periodo de tiempo de 4 años, el alcance fue correlacional, puesto que se determinó el coeficiente de correlación para establecer si existe relación entre el capital intelectual y la generación de valor a través del EVA. Se utilizó el método de caso simple para lo cual se obtuvieron datos de los estados financieros de los informes anuales de la empresa del sector tecnológico Samsung Electronics y otra información pública de los años de 2015 a 2018.

\section{Resultados}

\subsection{Descripción de la empresa y del sector}

De acuerdo con datos del INEGI (2018), el 82.4\% de la población está interesada en desarrollos científicos y tecnológicos y pese a que en los últimos años el sector industrial en México se ha estancado, la influencia de la situación global en cuanto al enorme desarrollo tecnológico, crea el interés de este estudio al analizar este sector puesto que promete un buen crecimiento y mayor importancia en los próximos años. Por otro lado las empresas tecnológicas son de las que más invierten en cuestiones de capital intelectual y en las que es muy visible el impacto de este en su valor.

Samsung Electronics es una empresa Sudcoreana que se dedica al negocio de la electrónica de consumo, tecnología de la información y comunicaciones móviles y soluciones de dispositivos en todo el mundo. Samsung Electronics Co., Ltd. fue fundada en 1938 y tiene su sede en Suwon, Corea del Sur (Bloomberg, 2019). 
A lo largo de su historia ha adquirido estratégicamente empresas que le han permitido abarcar un amplio sector. Por otro lado, también ha ofrecido una gran gama de productos, desde televisores a blanco y negro hasta productos como teléfonos inteligentes (smartphones), electrodomésticos o desarrollo de software, Ilevándola a ser una de las empresas que siempre está a la vanguardia, que es precursora en innovación tecnológica y una de las compañías más importantes de tecnología a nivel internacional.

En 2018 contaba con 309,630 empleados, 2,389 proveedores de primer nivel, abarcaba 74 ciudades con 216 centros operativos alrededor del mundo y un total de gastos en investigación y desarrollo (I + D) por 19 billones de Won coreanos.

En la figura 2 se observa el comportamiento de las ventas netas de Samsung del año 2015 al año 2018:

Figura 2. VENTAS NeTAS DE 2015 A 2018 (MILLONES DE USD)

VENTAS NETAS

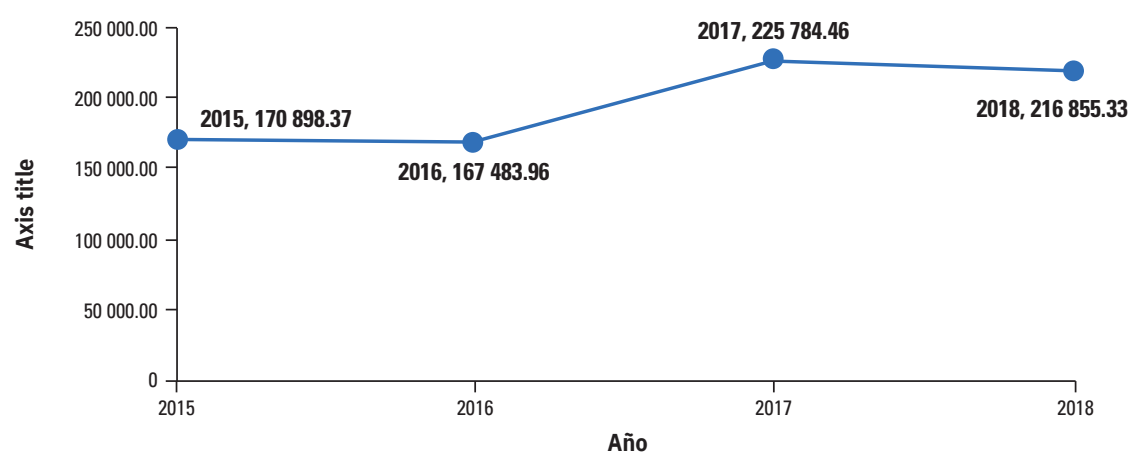

Fuente: elaborado con base en Samsung 2015, Samsung 2016, Samsung 2017, Samsung 2018.

Con base en el importe de ventas sabemos que Samsung es una empresa grande, comparada con otras empresas del mismo sector. Se decidió considerarla para realizar este estudio debido a la información disponible y a su historia de éxito dentro de su giro. Al realizar la búsqueda de información en empresas de este sector, principalmente en México, se detectó que muy pocas de estas brindan algún tipo de información ya sea financiera o de capital intelectual. 


\subsection{Aplicación del modelo Skandia}

De acuerdo con la metodología de cálculo del modelo Skandia, en el cuadro 6, se presenta la determinación del valor del capital intelectual en términos monetarios.

Cuadro 6. Determinación del VAlor del CAPital intelectual (Millones de USD)

\begin{tabular}{|c|c|c|c|c|c|}
\hline $\begin{array}{l}\text { No. } \\
\text { Indicador }\end{array}$ & Capital o Enfoque Humano & 2018 & 2017 & 2016 & 2015 \\
\hline & $\begin{array}{l}\text { Total de gastos en sueldos y } \\
\text { salarios }\end{array}$ & 20,218 & 21,092 & 15,987 & 15,643 \\
\hline 1 & $\begin{array}{l}\text { Proporción de empleados } \\
\text { menores de } 40 \text { años (\%) }\end{array}$ & 0.8293 & 0.8505 & 0.8603 & 0.8740 \\
\hline 2 & Rotación de empleados (\%) & 0.2240 & 0.1940 & 0.2240 & 0.2090 \\
\hline 3 & Tiempo de formación (\%) & 0.0071 & 0.0084 & 0.0081 & 0.0089 \\
\hline 4 & Hombres (\%) & 0.5700 & 0.5500 & 0.5600 & 0.5400 \\
\hline 5 & Mujeres (\%) & 0.4300 & 0.4500 & 0.4400 & 0.4600 \\
\hline \multirow[t]{5}{*}{6} & $\begin{array}{l}\text { Satisfacción de los empleados } \\
(\%)\end{array}$ & 0.9310 & 0.9640 & 0.9500 & 0.9200 \\
\hline & Total índices Capital Humano & 2.9914 & 3.0169 & 3.0424 & 3.0119 \\
\hline & Capital estructural & & & & \\
\hline & Inversión total en activos & 26,152 & 40,917 & 21,152 & 21,733 \\
\hline & Enfoque clientela & & & & \\
\hline 7 & $\begin{array}{l}\text { Satisfacción del cliente } \\
\text { (puntos) }\end{array}$ & 0.8540 & 0.8390 & 0.8400 & 0.8370 \\
\hline \multirow[t]{2}{*}{8} & $\begin{array}{l}\text { Empleados certificados con } \\
\text { trayectoria profesional de } \\
\text { servicio al cliente (\%) }\end{array}$ & 0.9300 & 0.9580 & 0.9500 & 0.9700 \\
\hline & Proveedores & & & & \\
\hline \multirow[t]{2}{*}{9} & $\begin{array}{l}\text { Proveedores con calificación } \\
\text { excelente (\%) }\end{array}$ & 0.6400 & 0.6000 & 0.7000 & 0.7000 \\
\hline & $\begin{array}{l}\text { Total índices de Capital } \\
\text { estructural }\end{array}$ & 2.4240 & 2.3970 & 2.4900 & 2.5070 \\
\hline $\mathrm{n}$ & $\begin{array}{l}\text { Total Capital humano y Capital } \\
\text { estructural }\end{array}$ & 5.4154 & 5.4139 & 5.5324 & 5.5189 \\
\hline$x$ & Número de indicadores & 9.0000 & 9.0000 & 9.0000 & 9.0000 \\
\hline \multirow[t]{2}{*}{$\mathrm{i}=(\mathrm{n} / \mathrm{x})$} & Coeficiente de eficiencia & 0.6017 & 0.6015 & 0.6147 & 0.6132 \\
\hline & $\begin{array}{l}\text { Total Capital humano y } \\
\text { Capital estructural (unidades } \\
\text { monetarias) }\end{array}$ & 46,370 & 62,009 & 37,139 & 37,377 \\
\hline$C I O=i * c$ & Valor del Capital Intelectual & 27,901 & 37,301 & 22,829 & 22,920 \\
\hline
\end{tabular}

Fuente: Elaborado con base en los datos de Samsung 2015, Samsung 2016, Samsung 2017, Samsung 2018. 
En 2015 el valor del capital intelectual fue de 22,920 millones de dólares americanos, en el año 2016 el valor del capital intelectual fue de USD 22,829 millones, esto debido a una mayor inversión en la parte del capital humano; pero el mayor aumento en el valor del capital intelectual ocurrió en el año 2017 llegando a USD 37,302 millones impulsado por una mayor inversión en el capital estructural y en menor medida el aumento en el capital humano. Finalmente, para el año 2018 disminuyó considerablemente, alcanzando solamente los USD 27,901 millones, debido nuevamente a una menor inversión en el capital estructural pese a que se mantuvo el capital humano

\subsection{Aplicación del modelo EVA}

Para la aplicación del modelo EVA se obtuvo información de los estados financieros de Samsung Electronics, y datos tanto económicos como de mercado para determinar, la utilidad operativa después de impuestos, el capital invertido y el costo de capital promedio ponderado, Ilegando a los resultados que se presentan en el cuadro 7 Determinación del EVA :

Cuadro 7. Determinación del EVA (Millones de USD)

\begin{tabular}{lcccr}
\hline & AÑo & AÑo & AÑo & AÑo \\
\cline { 2 - 5 } & $\mathbf{2 0 1 8}$ & $\mathbf{2 0 1 7}$ & $\mathbf{2 0 1 6}$ & $\mathbf{2 0 1 5}$ \\
\hline $\begin{array}{l}\text { Utilidad Neta de Operación Ajustada Des- } \\
\text { pués Impuestos }\end{array}$ & 38,714 & 38,614 & 18,464 & 17,446 \\
\hline Capital Invertido & 342,289 & 316,975 & 258,774 & 238,137 \\
\hline Costo de Capital Promedio Ponderado & $6.53 \%$ & $8.11 \%$ & $8.19 \%$ & $7.39 \%$ \\
\hline EVA & 16,370 & 12,908 & $-2,733$ & -159 \\
\hline
\end{tabular}

Los resultados obtenidos para el año 2015 muestran que no se generó valor en ese año. Se destruyó valor, por USD 159 millones debido a la baja utilidad de operación, para 2016 se continuó destruyendo valor al obtener una cifra negativa por USD 2,733 millones pese a que la utilidad neta de operación después de impuestos fue mayor, también se incrementó el capital invertido derivado del incremento en el renglón de efectivo e inversiones a corto plazo como se puede observar en el balance general. Además, el costo de capital promedio ponderado fue más elevado durante el año 2016, impulsado por el incremento en los préstamos ban- 
carios, mayormente aquellos de largo plazo, como se reportó en las notas a los estados financieros.

En 2017 Samsung comienza a generar valor, como consecuencia de una mayor utilidad de operación. Finalmente, durante el año 2018 vuelve a incrementar el valor, aunque de forma más moderada. Los resultados anteriores indican que las estrategias de la empresa están dando resultados, pues, aunque en los primeros años del análisis destruyo valor posiblemente por una mayor inversión que aún no repercutía en las utilidades, en los dos últimos años la generación de valor es evidente.

\subsection{Aplicación del modelo VAIC}

De acuerdo con la literatura el cálculo se desarrollará con base en los pasos indicados por Villegas, Hernández y Salazar, (2017) utilizando los datos tomados de los estados financieros divulgados por la propia Samsung Electronics.

\section{VAIC $=I C E+C E E$}

Donde ICE es el índice de eficiencia de capital intelectual y CEE es el índice de eficiencia del capital físico y financiero.

Índice de eficiencia de capital intelectual: ice $=$ hce + sce + rce

Índice de eficiencia de capital humano: hce $=\mathrm{va} / \mathrm{hc}$

Valor agregado: va $=$ out - in

El resultado de este cálculo puede verse en el cuadro 8. Determinación del VAIC:

\section{CuAdro 8. Determinación del VAIC}

\begin{tabular}{|c|c|c|c|c|c|c|c|}
\hline Paso & Dato & Fórmula & $\begin{array}{l}\text { Varia- } \\
\text { bles }\end{array}$ & 2018 & 2017 & 2016 & 2015 \\
\hline \multirow{3}{*}{ Paso 1} & \multirow{3}{*}{$\begin{array}{l}\text { Cálculo del } \\
\text { valor añadido } \\
\text { (VA) }\end{array}$} & \multirow{3}{*}{$V A=I T-C V$} & $\mathrm{IT}=$ & 216,855 & 225,784 & 167,484 & 170,898 \\
\hline & & & $C V=$ & 117,776 & 121,848 & 99,792 & 105,171 \\
\hline & & & $V A=$ & 99,079 & 103,936 & 67,692 & 65,728 \\
\hline \multirow{4}{*}{ Paso 2} & $\begin{array}{l}\text { Cálculo del } \\
\text { coeficiente de }\end{array}$ & \multirow{4}{*}{$\begin{array}{l}\mathrm{HCE}=\mathrm{VA} / \\
\mathrm{HC}\end{array}$} & & & & & \\
\hline & $\begin{array}{l}\text { eficiencia del } \\
\text { capital humano }\end{array}$ & & $\mathrm{VA}=$ & 99,079 & 103,936 & 67,692 & 65,728 \\
\hline & & & $\mathrm{HC}=$ & 20,218 & 21,092 & 15,987 & 15,643 \\
\hline & (HCE) & & $\mathrm{HCE}=$ & 4.90 & 4.93 & 4.23 & 4.20 \\
\hline
\end{tabular}


CuAdro 8. Determinación del VAiC (CONTINUACIÓN)

\begin{tabular}{|c|c|c|c|c|c|c|c|}
\hline Paso & Dato & Fórmula & $\begin{array}{l}\text { Varia- } \\
\text { bles }\end{array}$ & 2018 & 2017 & 2016 & 2015 \\
\hline \multirow{3}{*}{ Paso 3} & $\begin{array}{l}\text { Cálculo del } \\
\text { coeficiente de }\end{array}$ & \multirow{3}{*}{$\begin{array}{l}\mathrm{SCE}=\mathrm{SC} / \\
\mathrm{VA}\end{array}$} & $\begin{array}{l}\mathrm{SC}=- \\
V A-\mathrm{HC}\end{array}$ & 78,861 & 82,844 & 51,705 & 50,084 \\
\hline & $\begin{array}{l}\text { eficiencia del } \\
\text { capital estruc- } \\
\text { tural }\end{array}$ & & $V A=$ & 99,079 & 103,936 & 67,692 & 65,728 \\
\hline & (SCE) & & $\mathrm{SCE}=$ & 0.80 & 0.80 & 0.76 & 0.76 \\
\hline \multirow{3}{*}{ Paso 4} & $\begin{array}{l}\text { Cálculo del } \\
\text { coeficiente de }\end{array}$ & \multirow{3}{*}{$\begin{array}{l}\mathrm{ICE}=\mathrm{S}- \\
\mathrm{CE}+\mathrm{HCE}\end{array}$} & $\mathrm{SCE}=$ & 0.80 & 0.80 & 0.76 & 0.76 \\
\hline & $\begin{array}{l}\text { eficiencia del } \\
\text { capital intelec- } \\
\text { tual }\end{array}$ & & $\mathrm{HCE}=$ & 4.90 & 4.93 & 4.23 & 4.20 \\
\hline & (ICE) & & $\mathrm{ICE}=$ & 5.70 & 5.72 & 5.00 & 4.96 \\
\hline \multirow{3}{*}{ Paso 5} & $\begin{array}{l}\text { Cálculo del } \\
\text { coeficiente de }\end{array}$ & \multirow{3}{*}{$\begin{array}{l}\text { CEE=VA/ } \\
C E\end{array}$} & $V A=$ & 99,079 & 103,936 & 67,692 & 65,728 \\
\hline & $\begin{array}{l}\text { eficiencia } \\
\text { del capital } \\
\text { empleado }\end{array}$ & & $\mathrm{CE}=$ & 301,887 & 284,382 & 217,520 & 206,266 \\
\hline & (CEE) & & CEE $=$ & 0.33 & 0.37 & 0.31 & 0.32 \\
\hline \multirow{3}{*}{ Paso 6} & $\begin{array}{l}\text { Cálculo de } \\
\text { coeficiente de } \\
\text { valor }\end{array}$ & $\begin{array}{l}\text { VAIC=I- } \\
C E+C E E\end{array}$ & $\mathrm{ICE}=$ & 5.70 & 5.72 & 5.00 & 4.96 \\
\hline & $\begin{array}{l}\text { añadido inte- } \\
\text { lectual (VAIC) }\end{array}$ & & $\mathrm{CEE}=$ & 0.33 & 0.37 & 0.31 & 0.32 \\
\hline & & & VAIC $=$ & 6.02 & 6.09 & 5.31 & 5.28 \\
\hline
\end{tabular}

Con base en los valores de referencia proporcionados por Demuner, Saavedra y Camarena, (2017) en el cuadro 9 se observa lo siguiente:

\section{CuAdro 9. VAlores de los COMPONENTES DE VAIC}

\begin{tabular}{lrrrrl}
\hline & $\mathbf{2 0 1 8}$ & $\mathbf{2 0 1 7}$ & $\mathbf{2 0 1 6}$ & $\mathbf{2 0 1 5}$ & \\
\hline ICE & 5.70 & 5.72 & 5.00 & 4.96 & $\geq 2.5$ \\
\hline HCE & 4.90 & 4.93 & 4.23 & 4.20 & $\geq 2$ \\
\hline SCE & 0.80 & 0.80 & 0.76 & 0.76 & $\geq 0.5$ \\
\hline
\end{tabular}

El Îndice de eficiencia en el Capital Intelectual en cada uno de los años supera el valor de referencia por lo que se deduce primeramente que tiene un desempeño exitoso, sobre todo se nota que la empresa sigue siendo constante en su 
esfuerzo por mejorar ya que el índice mostró un incremento desde el año 2015 hasta el año 2018. De 2015 a 2017 se incrementó el ICE en 0.78 puntos y aunque bajó 0.2 para 2018 es mucho más considerable el incremento que la pequeña baja, en el período de análisis.

El índice de eficiencia de capital humano mostró una tendencia similar al ICE y cada año se incrementó hasta que tuvo una pequeña baja en 2018, por su parte el Índice Estructural se mantuvo constante y con un ligero incremento. Los resultados indican que el capital humano aporta más a la eficiencia de la empresa, aproximadamente entre $85 \%$ y $86 \%$ y que el capital estructural representa entre $14 \%$ y $15 \%$. De ahí la importancia de administrar correctamente el capital humano, a fin de mantener y mejorar la eficiencia de su uso.

\subsection{Análisis correlacional}

Se determinó el coeficiente de correlación a utilizar, mediante el uso del sistema SPSS Statistics Versión 20, para saber si se presentaba normalidad en las variables utilizando la prueba de Kolgomorov-Smirnov, Ilegando a la conclusión de utilizar el coeficiente de correlación se Spearman debido a que no se presentó normalidad en las variables. Posteriormente se realizó el cálculo mediante el mismo sistema cuyos resultados se observan en el cuadro 10. Correlación del capital intelectual con el EVA.

CuAdro 10. Correlación del CAPITAL INTElECtual Con El EVA

\begin{tabular}{lrrrr}
\hline Año & Valor del CI & EVA & $\begin{array}{r}\text { Coeficiente de Correlación } \\
\text { de Spearman }\end{array}$ \\
\hline 2015 & 22,920 & -159 & 0.80 \\
\hline 2016 & 22,830 & $-2,733$ & \\
\hline 2017 & 37,302 & 12,908 & \\
\hline 2018 & 27,902 & 16,370 & \\
\hline
\end{tabular}

Los resultados arrojaron que la correlación entre el valor del capital intelectual y el EVA es positiva y fuerte, arrojando un coeficiente de 0.80 , es decir, que existe relación entre el capital intelectual y el valor generado por la empresa. Por lo que será necesario impulsar el capital intelectual con el fin de mejorar el valor de la empresa. 
También, se realizó el cálculo del coeficiente de correlación entre el valor monetario del capital intelectual y eI VAIC (transformando los valores a logaritmos). Para establecer el modelo de correlación, se determinó (mediante el uso del sistema SPSS Statistics Versión 20), si se presentaba normalidad en las variables, Ilegando a la conclusión de utilizar el coeficiente de correlación se Spearman debido a que no se presentó normalidad en las variables y obteniendo un coeficiente de correlación de 0.80 , es decir, una correlación positiva fuerte. Lo que indica que el valor agregado y la eficiencia están íntimamente relacionados con el valor del capital intelectual.

CuAdRo 11. CoRRELACIÓn DEL CAPITAL INTELECTUAL CON EL VAIC

\begin{tabular}{lrrrr}
\hline Año & Log CI & Log VAIC & $\begin{array}{r}\text { Coeficiente de correlación de } \\
\text { Spearman }\end{array}$ \\
\hline 2015 & 4.36 & 0.72 & 0.80 \\
\hline 2016 & 4.36 & 0.73 & \\
\hline 2017 & 4.57 & 0.78 & \\
\hline 2018 & 4.45 & 0.78 & \\
\hline
\end{tabular}

\section{Conclusiones}

Samsung, es una organización muy bien posicionada en el mercado mundial lo que le da ventaja competitiva y lo que la ubica en indicadores por encima del sector, es una empresa que, con base en sus datos financieros y no financieros, tiene una gran estructura y alcance que le dan buenos resultados. El análisis cuantitativo del capital intelectual de Samsung, brindó datos valiosos, pero no es concluyente para el sector puesto que, aunque es una empresa suficientemente grande, se encuentra en una posición diferente a la de sus competidores. Sin embargo, esos valiosos datos nos son útiles para entender cómo funciona el capital intelectual en empresas de este tipo, dado que nos muestra que la correcta gestión de este, puede dar beneficios satisfactorios, así como también demuestra que el éxito de una empresa depende tanto de lo financiero como de lo no financiero en este caso del capital intelectual y por lo tanto debe dársele la importancia que corresponde a cada uno de estos. El trabajo empírico con esta empresa demostró que es posible determinar el valor del capital intelectual utilizando el modelo Skandia. 
En este trabajo de investigación se utilizaron los modelos VAIC y EVA, ambos para determinar la eficiencia de los recursos e identificar la generación de valor, no solo si lo genera y cuánto, sino también saber qué elementos son los que generan valor y en qué medida. Se determinó una importante relación entre VAIC y el valor del capital intelectual, el primero como un indicador de eficiencia de uso de todos los recursos que componen el capital intelectual, demostrando así la importancia de administrar correctamente el capital intelectual para obtener eficiencia del uso del mismo. También, se logró establecer una relación importante entre el EVA y el valor del capital intelectual, demostrando con esto la importancia de administrar correctamente el capital intelectual para generar valor en la empresa.

El principal aporte de este trabajo, es demostrar a través de una aplicación empírica el uso y bondades de 3 modelos Skandia, VAIC y EVA, que aunque son muy conocidos son poco utilizados por los analistas de valores, tal vez por la limitación en obtener información específica acerca del capital intelectual de las empresas, pues en el contexto mexicano no están obligadas a reportarlo, otra limitación se debería al desconocimiento de la metodología para su cálculo, lo cual se logró establecer en este trabajo. Una limitación importante de esta investigación es el uso del método de caso simple, lo cual no permite generalizar los resultados y se deben tomar con sumo cuidado los hallazgos.

\section{Bibliografía}

Adam, J. (2005). Los métodos de valuación de empresas y su relación con la capacidad de las organizaciones para generar valor. Propuesta para reportar en la información financiera el valor de las organizaciones y su capacidad para generarlo. Contaduría y Administración(217), 11-47.

Arango, M., Molina, P., y Zapata, J. (septiembre-diciembre de 2010). Revisión de metodologías para la valoración del capital intelectual organizacional. Revista Virtual Universidad Católica del Norte(31), 105-130.

Archibold, W., y Escobar, A. (2015). Capital intelectual y gestión del conocimiento en las contralorías territoriales del departamento del atlantico. Revista Dimensión Empresarial, 13(1), 133-146. doi:: http://dx.doi.org/10.15665/rde.v13i1.342

Bloomberg, L.P. (s.f.). www.bloomberg.com. Recuperado el 23 de 07 de 2019, de https:// www.bloomberg.com/research/stocks/private/snapshot.asp?privcapld=91868 
Bontis, N. (Marzo de 2001). Assessing Knowledge assets: a review of the models used to measure intellectual capital. Intenational Journal of Management Reviews, 3(1), 41-60.

Brooking, A. (1997). El capital Intelectual. El principal activo de las empresas del tercer milenio. Barcelona: Paidós Iberica.

Cazares, L., Saavedra, M. y Tapia, B. (2019). Creación de valor en la industria cervecera artesanal mexicana. Revista Economía, XLIV, 47 (enero-junio, 2019), 101-130.

Cegarra, J., y Jiménez, J. (2002). Capital Intelectual: Aproximación al sector curtidos de la región murcia. Cuadernos de Economía Murciana: revista de economia regional(15), 17-34.

Demuner, M., Saavedra, M., y Camarena, M. (2017). Medición del capital intelectual en el sector bancario: aplicación de los modelos Skandiay VAIC. Innovar, 27(66), 75-89.

Edvinsson, L., y Malone, M. (1997). Intellectual capital: realizing your company's true calue by finding its hidden roots (1a Edición ed.). New York: HarperCollins Publishers, inc.

Conzález, J. (Junio de 2009). El capital intelectual y sus indicadores en el sector industrial. (U. N. Colombia, Ed.) TEACS, 01(02), 9-33.

Hernando, C., y Cervera, M. (Enero-Junio de 2018). Influencia del capital intelectual en la gestión de la pequeña empresa familiar española. Un estudio empirico. RICC, XVI(31), 1-17.

Instituto Nacional de Estadística y Ceografía. (2018). Encuesta nacional sobre productividad y competitividad de las micro, pequeñas y medianas empresas /ENAPROCE) 2018. México. Recuperado el 27 de 11 de 2019, de https://www.inegi.org.mx/ programas/enaproce/2018/

Joya, R., Gámez, L., Ortíz, M., y Gálvez, A. (2015). Medición del capital intelectual en empresas mexicanas. Retos de la Dirección, 9(1), 79-95.

Kristandl, G., y Bontis, N. (2007). Constructing a definition for intangibles using the resource based view of the firm. Management Decision, 45(9), 1510-1524. doi: $10.1108 / 00251740710828744$

Laing, G., Dunn, J., y Hughes, S. (2010). Applying the VAIC model to Australian hotels. Journal of intellectual capital, 11(3), 269-283.

Larios, P. (2010). El Capital Intelectual como generador de valor y ventaja competitiva en los inicios del nuevo milenio. México, DF: Universidad Nacional Atónoma de México. Mena, C., Vásconez, H., y Carguaytongo, J. (Agosto de 2017). El capital intelectual desde una revisión teórica de la literatura publicada. Dominio de las Ciencias, 3 , 29-50. 
Nevado, D., y López, V. (2016). Modelo de control estrátegico desde la perspectiva del valor de los intangibles. Método y aplicación. Revista Innovar Journal, 25(59), 9-20. doi:http://dx.doi.org/10.15446/innovar.v26n59.54319

Saavedra, G. (2004). La valuación de empresas en México: Aplicación del modelo de valor económico agregado 1991-2000. Revista Contaduría y Administración, 214, pp.1-23.

Saavedra, M., y Saavedra, M. (Julio-Diciembre de 2012). Una propuesta de medición e incorporación del capital intelectual en la información financiera: el caso de Unión Febre. Cuadernos de contabilidad, 13(33), 505-526.

Samsung Electronics Inc. (2015). Reporte Estados Financieros. Obtenido de Samsung. com.

Samsung Electronics Inc. (2016). Reporte Estados Financieros. Obtenido de Samsung. com.

Samsung ELectronics Inc. (2017). Reporte Estados Financieros. Obtenido de Samsung. com.

Samsung Electronics Inc. (2018). Reporte Estados Financieros. Obtenido de Samsung. com.

Sarur, M. (2013). La importancia del capital intelectual en las Organizaciones. Ciencia Administrativa(1), 39-45.

Steward, T. (1997). Intellectual Capital; The New Wealth of Organizations. New York: Doubleday/Currency.

Sveiby, K. (1997). The Intangible Assets Monitor. Journal of human resource costing and accounting, 2(1), 73-97.

Ureña, Y., Quiñoes, E., y Carruyo, N. (2016). Capital Intelectual: Modelo estratégico para la calidad de servicio en las organizaciones inteligentes. Revista Científica Electrónica de Ciencias Humanas, 12 (35), 3-17.

Villacorta, M. (2003). Revelación de la información voluntaria sobre el Capital Intelectual en los informes anuales de las empresas cotizadas. Cuadernos de estudios empresariales(13), 365-391.

Villegas, E., Hernández, M., y Salazar, B. (2017). La medición del capital intelectual y su impacto en el rendimiento financiero en empresas del sector industrial en México. Contaduría y Administración, 62, 184-206. 